\title{
Características relevantes de la simulación energética de viviendas unifamiliares
}

\section{Critical features of energy simulation for single housing}

\author{
$\underline{\text { R. García-Alvarado }}^{(*)}$, A. González ${ }^{(*)}$, W. Bustamante ${ }^{(* *)}$, A. Bobadilla ${ }^{(*)}$, C. Muñoz ${ }^{(*)}$
}

\section{RESUMEN}

Los sistemas de simulación energética de edificios permiten analizar la calidad y demanda ambiental de las construcciones, pero deben contar con procedimientos fiables y pertinentes. Este artículo identifica condiciones relevantes de la simulación energética de viviendas unifamiliares, basado en el análisis de una docena de programas y distintas experiencias en el centro sur de Chile. Desarrollando una revisión de capacidades de los sistemas, a través de la simulación de una misma vivienda y entrevistas a usuarios expertos. Además de estudiar incidencias de los antecedentes y distintas estrategias de análisis para el mejoramiento ambiental de viviendas. Revelando características significativas para el estudio energético de construcciones habitacionales aisladas. Finalmente sugiere revisar ciertas condiciones de los sistemas para asegurar la efectividad de los análisis ambientales y de las acciones subsecuentes.

Palabras clave: Simulación energética; vivienda unifamiliar; construcción sustentable; Chile.

\section{SUMMARY}

Energy simulation systems allow analyzing the environmental quality and demand of buildings, but they must have reliable and proper procedures. This paper identifies relevant conditions of energy simulation systems for the study of single-family homes, based on the analysis of a dozen programs and various experiences in south central Chile. A review of capabilities was conducted, simulating the same case in all systems and making interviews with expert users. Besides it studied the incidence of background data and different strategies for analysis the environmental performance of housing. Revealing significant characteristics in the energy study of detached residential buildings. Finally it suggests reviewing certain conditions to ensure the effectiveness of the environmental analysis and subsequent actions.

Keywords: Energy simulation; single housing; sustainable building, Chile.

(*) Universidad del Bío-Bío. Concepción (Chile).

(**) Pontificia Universidad Católica de Chile. Santiago (Chile).

Persona de contacto/Corresponding author: rgarcia@ubiobio.cl (R. García-Alvarado)

Cómo citar este artículo/Citation: García-Alvarado, R., González, A., Bustamante, W., Bobadilla, A., Muñoz, C. (2014). Características relevantes de la simulación energética de viviendas unifamiliares. Informes de la Construcción, 66(533): e005, doi: http://dx.doi.org/10.3989/ic.12.108.

Licencia / License: Salvo indicación contraria, todos los contenidos de la edición electrónica de Informes de la Construcción se distribuyen bajo una licencia de uso y distribución Creative Commons Reconocimiento no Comercial 3.o. España (cc-by-nc). 


\section{INTRODUCCIÓN}

El progresivo agotamiento de las fuentes energéticas tradicionales y los requerimientos crecientes de habitabilidad, están promoviendo diversas acciones para mejorar el desempeño sostenible de las construcciones. Para asegurar la calidad ambiental y reducir el consumo energético de los edificios, se requiere un adecuado análisis de su comportamiento. Se dispone en la actualidad de una amplia variedad de sistemas de simulación energética, que todavía presentan limitaciones importantes que se analizan en el presente trabajo. Lo anterior especialmente en pequeñas edificaciones, como las viviendas unifamiliares, que se sitúan en diferentes contextos climáticos y muestran diferentes estándares constructivos.

El presente artículo propone identificar condiciones relevantes de los análisis energéticos para residencias individuales, basados en diversas experiencias en el centro-sur de Chile, en donde se ha efectuado una progresiva regulación de su situación habitacional, que puede ilustrar su aplicación para distintos países en desarrollo.

En Chile las construcciones residenciales son responsables de más de la mitad de los gastos energéticos del sector de la edificación, con un 77\% el año 2011 en todos los servicios del área Comercial-Público-Habitacional (1), incluyendo conjuntos multifamiliares o pequeñas viviendas unifamiliares. Las residencias individuales poseen distintas formas, localizaciones y ocupaciones, que requieren un estudio particular. Por su reducido tamaño, se deben realizar análisis breves y eficaces, por lo que las simulaciones energéticas de viviendas unifamiliares deben reconocer aspectos significativos para sostener estudios adecuados.

\subsection{Sistemas de Simulación Energética}

Los procedimientos de cálculo energético de las edificaciones emergieron a partir de la crisis del petróleo en los años 70, y en las últimas décadas se han implementado en diversos sistemas computacionales, aprovechando sus capacidades de procesamiento matemático y representación gráfica (Figura 1). Se disponen en la actualidad de una variedad de programas gratuitos o comerciales para simulación energética de edificio. El Departamento de Energía de los Estados Unidos mantiene desde 1996 un directorio de aproximadamente un centenar de herramientas computacionales (2), además de desarrollar algunas gratuitas, como Energy-Plus (originalmente denominado DOE), utilizado como motor de cálculo. En el directorio se encuentran descripciones y referencias de cada sistema, y varios están validados por las normas ISO 13790:2008-09 y ASRHAE 90.1 (3) (4). Algunos centros universitarios han elaborado programas de uso liberado, como ESP-r de la U.Strahclyde desde 1977, Trnsys desarrollado por la U. de Winscosin desde 1978, Archisum de la U. Politécnica de Catalunya desde 1992 o Casanova de la U. Siegen desde el 2000. Aunque la mayoría no mantiene una actualización regular o distribución amplia. Otros programas han surgido por legislaciones o certificaciones específicas, como LIDER en España, CCTE en Chile, CEPE en California y el PHPP para el estándar CasaPasiva de Alemania. También desarrolladores de productos, instaladores o investigadores independientes han elaborado algunas herramientas. Algunos aplican métodos simplificados o «estáticos» (sin variación cronológica), y otros son más completos o «dinámicos» (con registros por horas, días, semanas, etc.). Programas como ESP-r modelan el edificio por coordenadas, otros como TAS se basan en plantas 2D, Ecotect y Design Builder modelan en 3D, y algunos como Heed requieren sólo datos generales. Varios sistemas se pueden relacionar entre sí, permitiendo realizar la modelación con un programa, luego el cálculo en otro, y la revisión de datos por separado. Promoviendo la transferencia de archivos y la utilización encadenada de programas, pero generando un panorama diverso de herramientas posibles.

Recientemente proveedores importantes de software para diseño arquitectónico han elaborado o adquirido programas de análisis energético, (como Autodesk, con Ecotect y Vasari; Graphisoft con Ecodesigner, Nemetschek con Archiwizard, etc.), para facilitar la vinculación y comercialización conjunta. Así también han emergido servicios por internet que ofrecen analizar el comportamiento energético de edificios (como Green Building Studio de Autodesk o Design Advisor del MIT) o sistemas abiertos (como OpenStudio), pero no explican condiciones de trabajo adecuadas según el tipo de construcción o localización, por la falta de revisiones específicas, lo que se pretende aclarar en este artículo para viviendas unifamiliares en climas templados como el centro-sur de Chile. De este modo en la actualidad se puede encontrar una diversidad de sistemas (mayormente en idioma inglés), con distintas características y con utilización aún reducida en el ámbito profesional, especialmente en los países en desarrollo, debido a que recién se están incorporando conceptos de comportamiento energético en la formación académica, y en los procesos públicos o privados de diseño de edificios los consideran parcialmente. Pero presentan un requerimiento creciente para amplias acciones de mejoramiento ambiental de la construcción.
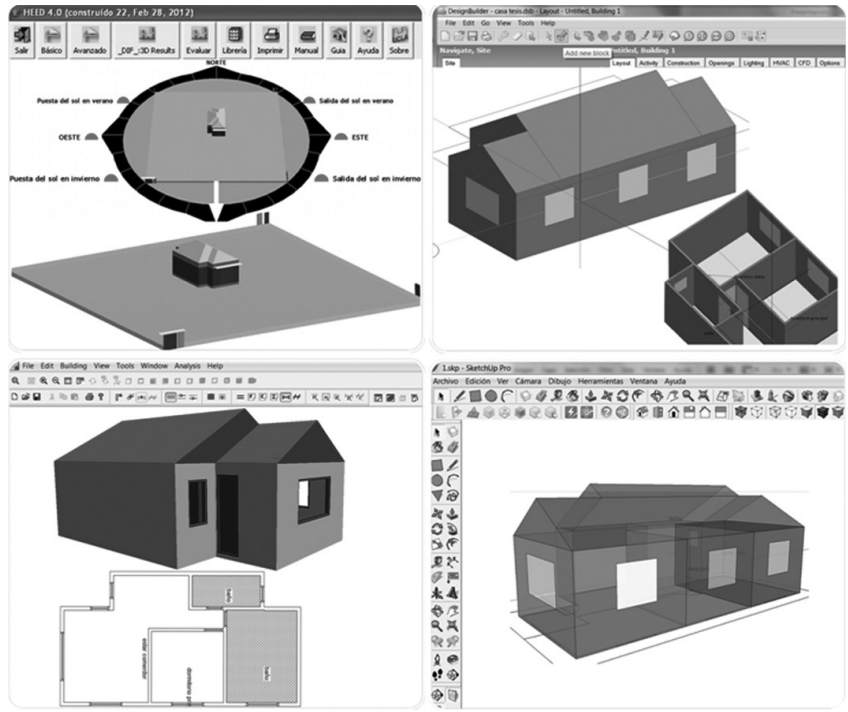

Figura 1. Distintos Software de Simulación Energética con el modelo de estudio.

\subsection{Proceso de Simulación Energética}

Los sistemas de análisis o simulación energética constituyen en general programas informáticos, en los cuales se deben indicar una variedad de características de los edificios, se calculan internamente sus transferencias térmicas (5), obteniendo resultados específicos que se exponen de diferentes maneras. Los antecedentes que se deben proveer se refieren a condiciones climáticas del lugar, forma de los recintos, vanos 
y entorno del edificio, materiales que lo componen, equipos instalados y patrones de ocupación. Basado en esta información se efectúan procesamientos matemáticos que indican las condiciones ambientales interiores (temperatura, humedad, calidad del aire, emisiones de $\mathrm{CO}_{2}$ ), y demandas energéticas por periodos. En algunos casos se expresan también características de radiación solar, iluminación natural, conducciones térmicas, circulación de aire, ciclo de vida de materiales o certificación ambiental. Con diversas modalidades de visualización gráfica o numérica, que pueden ser exportados a otros programas. Con los cuales se comparan y evalúan condiciones constructivas. La presentación y características de trabajo varían entre los diferentes sistemas, y las regulaciones o documentos no detallan mayormente las características o procedimientos. Esta indefinición puede generar una interpretación errada de los datos iniciales o resultados al momento de desarrollar un modelo predictivo.

Se han efectuado algunas evaluaciones comparativas de software (6) (7) (8) (9). Aunque no abarcan los más recientes, estos estudios revelan similitudes entre los sistemas y destacan diferencias fundamentalmente en la facilidad de utilización, en algunas capacidades y relación con otros software. Una propuesta de metodología para validar los programas (10), reconoce un funcionamiento regular en los cálculos de conducción térmica, así como diferencias en aspectos convectivos, y diversas incertidumbres en los datos ingresados. En revisiones del mismo caso utilizando diferentes software se han detectado hasta un 30\% de disparidad en los resultados de los mismos indicadores (11), atribuidos a ciertas consideraciones técnicas y situaciones locales. En otro estudio equivalente (12), se revelaron diferencias en los resultados mayormente por la complejidad de los edificios.

Las evaluaciones en general no consideran los sistemas más disponibles y se concentran en las capacidades internas, sin revisar condiciones de implementación (costos, instalación, soporte, formación de los usuarios), como tampoco de aplicación. Varias inquietudes frecuentes no son mencionadas, como sí las diferencias de precio o gratuidad entre los programas son relevantes; sí requieren conocimientos especializados; cuál es la incidencia de los antecedentes; cuánto esfuerzo se debe desarrollar en la implementación o análisis; si hay relación con el tipo de edificación o mejoramientos posibles; cómo se discriminan las distintas alternativas; como se integran en el diseño y la ejecución; y como se ponderan las incidencias económicas. Quedando entonces varios cuestionamientos relevantes para su aplicación.

\subsection{Desarrollo del estudio}

Con el fin de esclarecer estos aspectos se revisó una docena de software y servicios web de análisis energético de edificaciones, instalando cada sistema y efectuando con cada uno la simulación de una misma vivienda, además de consultar usuarios experimentados y bibliografía especializada, para desarrollar una valoración sintetizada. También, se revisaron distintos estudios energéticos de construcciones habitacionales en la zona, analizando la incidencia de sus antecedentes, y se efectuaron en particular simulaciones de una docena de viviendas existentes, con un mismo sistema y procedimientos. Realizando en estos casos, además un registro detallado de las condiciones constructivas, funcionales y ambientales, que se compararon con los modelos simulados. Se desarrollaron luego ajustes sucesivos para representar el desempeño real de las viviendas, sintetizando las adecuaciones realizadas. De este modo se arribó a una clasificación y focalización de sistemas apropiados para su aplicación en viviendas unifamiliares de la zona, identificando consideraciones significativas para los estudios energéticos de estas edificaciones.

\section{CONDICIONES DE LOS SISTEMAS DE SIMULACIÓN}

\subsection{Revisión de Capacidades}

Para revisar características de los sistemas, primero se consultó a especialistas y en publicaciones, los programas más relevantes y utilizados actualmente. Encontrando bastantes convergencias en los mencionados, y algunos recientes en software libre y servicios web, que pueden tener una proyección amplia. Se buscó programas revisados en evaluaciones anteriores, pero varios no estaban disponibles, lo que expresa un desarrollo relevante en los últimos años. La nómina final (Tabla 1) fue confirmada consiguiendo o adquiriendo los programas correspondientes, que se instalaron en computadores personales. Verificando entonces que los sistemas analizados fueran pertinentes y se pudieran utilizar adecuadamente. Algunos presentaron dificultades en este proceso, especialmente con los sistemas gratuitos, que carecen de servicio técnico o presentan detalles de instalación sin antecedentes. En un par de casos (IES-VE y DesignBuilder), existen versiones con distintas capacidades, y se eligió una comparable con las restantes. Otros funcionan combinados (DesignBuilder y OpenStudio operan con Energy Plus). De este modo se seleccionó una docena de programas, incluyendo algunos ampliamente difundidos, vinculados a grandes proveedores de software o instituciones en el área, como otros más nuevos e independientes.

Se probó el funcionamiento de todos los sistemas instalados, y además se entrevistó a usuarios experimentados y se efectuó con cada programa una simulación del mismo modelo. Los usuarios consultados (Tabla 2) fueron ubicados contactando las principales oficinas o instituciones que realizan análisis energético de edificios dentro del país. Se les realizo un cuestionario y también visitas directas, algunos en varias oportunidades. Consultando finalmente a una decena de profesionales, que poseían al menos dos años de trabajo en simulación energética de edificios, y que se desempeñaban en distintos centros de estudio y oficinas privadas. Las experiencias abarcaron sólo algunos de los programas, debido a que en varios sistemas nuevos o más específicos no se utilizan ampliamente, lo que expresa una concentración de las herramientas. La mayoría manifestó ocupar principalmente un sólo sistema, usualmente aprendido en un postgrado en el extranjero, y aunque estaban enterados de otros software, desconocían sus características. Se advirtió un predominio reciente de Ecotect, aunque los profesionales más experimentados utilizaban otros programas. Se les consulto fundamentalmente su apreciación de los software y su proceso de trabajo, solicitando mostrar algunos ejemplos y discutir su aplicación en viviendas unifamiliares.

La simulación realizada con todos los sistemas consistió en una pequeña vivienda aislada rectangular de un piso, que totalizaba una superficie de $60 \mathrm{~m}^{2}$, con muros de albañilería de ladrillos y piso de hormigón sobre el terreno (sin ventilación), ventanas de vidrio simple con marcos metálicos, estructura de techumbre de madera con cubierta metálica y aislamiento 
Tabla 1.

Sistemas revisados de Simulación Energética (en 2012).

\begin{tabular}{|c|c|c|c|c|c|}
\hline & Programa/Servicio & $\begin{array}{l}\text { Año de } \\
\text { Inicio }\end{array}$ & Proveedor & $\begin{array}{c}\text { Costo } \\
\text { (dólares) }\end{array}$ & Características Principales \\
\hline 1 & ESP-R & 1977 & U. Strathclyde & o & $\begin{array}{l}\text { Gran capacidad de calculo y detalles avanzados, funciona en } \\
\text { Windows, Mac y Linux }\end{array}$ \\
\hline 2 & TAS & 1984 & EDSL & 7.000 & $\begin{array}{l}\text { Modelación precisa y análisis detallado, preferido por } \\
\text { especialistas }\end{array}$ \\
\hline 3 & IES-VE (VE-WARE) & 1994 & IESVE & 61 a 9.000 & $\begin{array}{l}\text { Buen cálculo hasta } 5.000 \text { zonas, con distintas versiones, } \\
\text { permite modelar con Sketchup }\end{array}$ \\
\hline 4 & CASANOVA & 2000 & U. Siegen & o & $\begin{array}{l}\text { Fácil de utilizar y cálculos rápidos, pero de una sola zona y } \\
\text { volumen simple }\end{array}$ \\
\hline 5 & HEED & 2003 & UCLA & o & $\begin{array}{l}\text { Funcionamiento intuitivo, sin modelación, se enfoca en } \\
\text { análisis costo-beneficio de viviendas }\end{array}$ \\
\hline 6 & DESIGNBUILDER con E+ & 2005 & DesignBuilder Co. & 308 a 4.187 & $\begin{array}{l}\text { Cálculos avanzados usando Energy-Plus, con funcionamiento } \\
\text { intuitivo y simple. }\end{array}$ \\
\hline 7 & ECOTECT & 2008 & Autodesk & 800 a 1.200 & $\begin{array}{l}\text { Fácil modelación, visualización y análisis, buena exportación } \\
\text { y muy utilizado }\end{array}$ \\
\hline 8 & $\begin{array}{l}\text { GREEN BUILDING } \\
\text { STUDIO }\end{array}$ & 2008 & Autodesk & 400 & $\begin{array}{l}\text { Servicio de internet que permite ingresar archivos en formato } \\
\text { gbxml y cálculos rápidos }\end{array}$ \\
\hline 9 & OPEN STUDIO con E+ & 2008 & NREL & o & Permite modelar con Sketchup, calcula con Energy Plus \\
\hline 10 & VASARI & 2010 & Autodesk & o & $\begin{array}{l}\text { Cálculos sencillos pero variados y específicos de distintos } \\
\text { volúmenes, exporta desde Revit }\end{array}$ \\
\hline 11 & BEOPT con $\mathrm{E}+$ & 2011 & NREL & o & $\begin{array}{l}\text { Buen cálculo y asistencias, compara alternativas con análisis } \\
\text { paramétrico }\end{array}$ \\
\hline 12 & DESIGN ADVISOR & 2012 & MIT & o & Servicio de internet eficaz y completo \\
\hline
\end{tabular}

Tabla 2.

Lista de Usuarios Consultados.

\begin{tabular}{|c|l|l|l|c|}
\hline & \multicolumn{1}{|c|}{ Formación Académica } & \multicolumn{1}{c|}{ Dedicación } & Programa Utilizado & Años de Experiencia \\
\hline 1 & Magister en Arquitectura Sustentable & Arquitecto & Ecotect & 4 \\
\hline 2 & Diplomado en Arquitectura Sustentable & Arquitecto & Ecotect & 3 \\
\hline 3 & $\begin{array}{l}\text { Magister en Eficiencia Energética y Hábitat } \\
\text { Sustentable }\end{array}$ & Arquitecto & Ecotect & 5 \\
\hline 4 & $\begin{array}{l}\text { Magister en Medio Ambiente y Arquitectura } \\
\text { Bioclimática }\end{array}$ & Arquitecto & Design Builder, IES-VE & 3 \\
\hline 5 & $\begin{array}{l}\text { Magister en Medio Ambiente y Arquitectura } \\
\text { Bioclimática }\end{array}$ & Arquitecto & TAS & 5 \\
\hline 6 & Ingeniero en Construcción & Ingeniero en Construcción & TAS & 6 \\
\hline 7 & Magister en Ingeniería & Ingeniero Civil & Openstudio & 3 \\
\hline 8 & Magister en Arquitectura y Energías Renovables & Arquitecto & ESP-r & 3 \\
\hline
\end{tabular}

ligero, Lo que corresponde a la tipología de vivienda más frecuente en el país y la zona (en un 85\%), con el sistema constructivo más utilizado (en un $45 \%$, el restante corresponde a estructuras en madera o bloques), y la media de superficie (13), por lo que constituye un ejemplo representativo. Se consideró una ocupación regular de cinco personas, con una renovación de aire/hora por recinto y localización en la ciudad Concepción (latitud $36^{\circ} 46^{\prime}$, longitud $73^{\circ}$ o3'), que posee clima templado-húmedo y un rango de temperaturas durante el año entre 5 a $23^{\circ}$ (Figura 2). Se modelo la vivienda en cada programa, y en algunos casos se importaron los planos digitales como referencia (Figura 3). Utilizando las mismas condiciones climáticas, materiales y de ocupación (Tabla 3). $\mathrm{El}$ proceso fue realizado por un mismo usuario, obteniendo diferencias hasta de un 50\% en los resultados de demandas energéticas (Figura 4). Las mayores oscilaciones se presentaron en los programas gratuitos, mientras los sistemas paga- dos en general difirieron sólo en un 15\%. Luego se verificaron las incidencias de los distintos antecedentes en los sistemas, advirtiendo que estos provienen mayormente de consideraciones de calidad constructiva (infiltraciones), estimaciones de confort (bandas de temperatura) o calendarios de climatización, mientras condiciones de modelación, especificación material (incluyendo detalles de vanos o perfiles), equipamientos u ocupación, que difieren entre los programas, presentan influencias menores. Por tanto las oscilaciones de resultados entre sistemas no inciden significativamente en los estudios, aunque igualmente se requiere la mayor precisión posible para verificar comportamientos.

\subsection{Valoración de Condiciones}

Basados en estas actividades, se valoraron características de los sistemas, ya sea aspectos mencionados en evaluacio- 


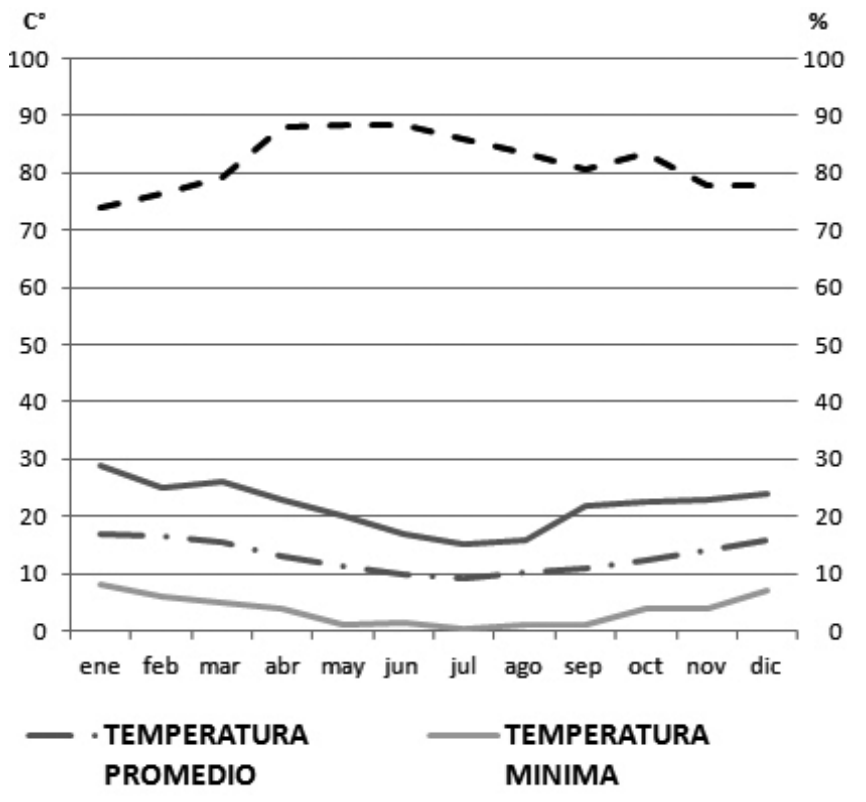

\section{-TEMPERATURA MAXIMA}

Figura 2. Clima de Concepción, Chile.

nes previas, como nuevas consideraciones de utilización e instalación. Algunas fueron cuantificadas, como rangos de costos o tiempos de procesamiento, existencia de ciertas operaciones, etc., y otras fueron calificadas subjetivamente, como presentación, utilidad, etc., según lo comentado por usuarios y apreciaciones comparativas. Utilizando una escala similar (de 1 a 5), con el valor más bajo para una situación negativa y el más alto, para una positiva. Las características se dividieron en dos grandes temas; capacidades técnicas y facilidad de ocupación (Figura 5). Debido a que la mayoría de las evaluaciones reconoce estas dos principales distinciones.

En la facilidad se contemplaron aspectos como la sencillez de modelación e ingreso de datos, menor tiempo de proceso,
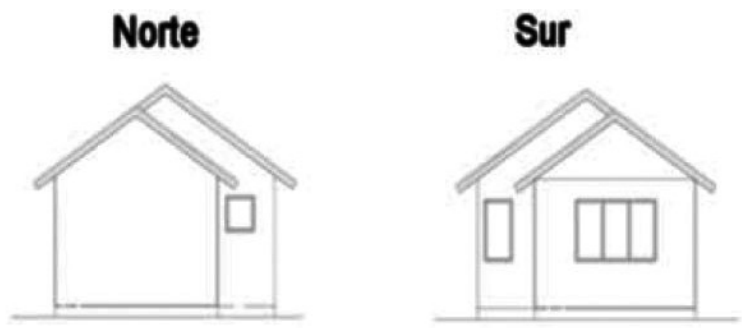

Este

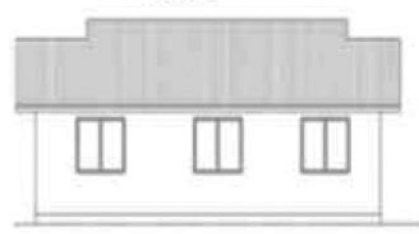

Oeste

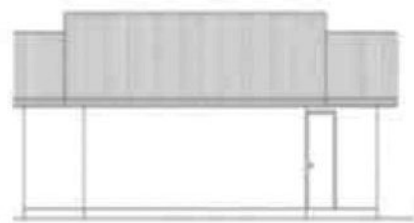

Planta

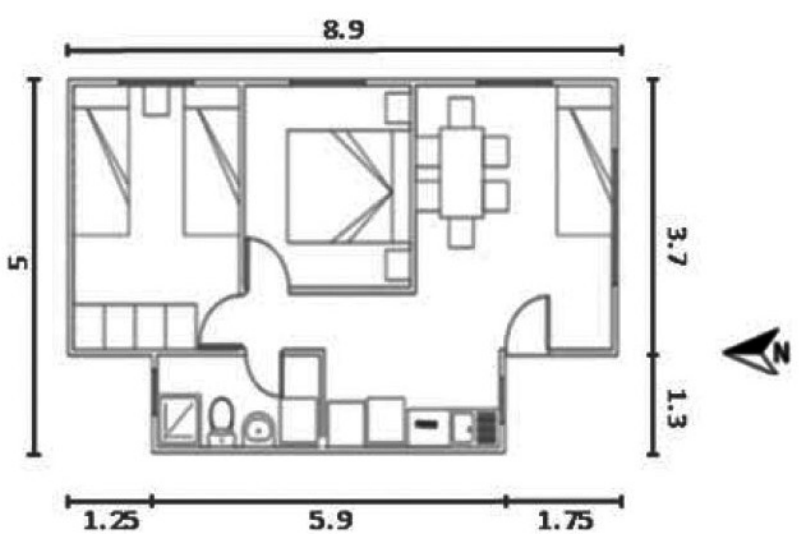

Figura 3. Vivienda Simulada

visualización de resultados, como también el precio (a menor costo, más facilidad de disponer el software), simplicidad de instalación, atención de servicio o información, menor requerimiento de equipos o formación de los usuarios, etc. En las capacidades, se consideraron características como la

Tabla 3.

Especificaciones y propiedades consideradas en la Vivienda Simulada.

\begin{tabular}{|c|c|c|c|}
\hline $\begin{array}{c}\text { Materialidad } \\
\mathbf{m m}\end{array}$ & \begin{tabular}{|c|}
$\begin{array}{c}\text { Densidad aparente } \\
\mathrm{kg} / \mathrm{m}^{3}\end{array}$ \\
\end{tabular} & $\begin{array}{c}\text { Conductividad térmica, } \\
\lambda W /(\mathbf{m} \times \mathbf{K})\end{array}$ & $\begin{array}{c}\text { resistencia térmica } \\
\mathbf{e} / \lambda \mathbf{m}^{2} \times \mathrm{K} / \mathrm{W}\end{array}$ \\
\hline \multicolumn{4}{|c|}{ Muro exterior } \\
\hline Ladrillo 140x240x71 & 1400 & 0,6 & 0,23 \\
\hline \multicolumn{4}{|c|}{ Tabiquería interior } \\
\hline Yeso cartón 8 mm & 700 & 0,26 & 0,03 \\
\hline Cámara de aire & - & - & - \\
\hline Yeso cartón $8 \mathrm{~mm}$ & 700 & 0,26 & 0,03 \\
\hline \multicolumn{4}{|c|}{ Cielo } \\
\hline Yeso cartón 10 mm & 700 & 0,26 & 0,038 \\
\hline Poliestireno $80 \mathrm{~mm}$ & 20 & 0,043 & 1,86 \\
\hline \multicolumn{4}{|c|}{ Techumbre } \\
\hline Plancha Zincalum 2 mm & 7130 & 112 & 1,78 \\
\hline \multicolumn{4}{|c|}{ Ventanas } \\
\hline Vidrio simple $6 \mathrm{~mm}$ & 2400 & 1,2 & 0,17 \\
\hline
\end{tabular}




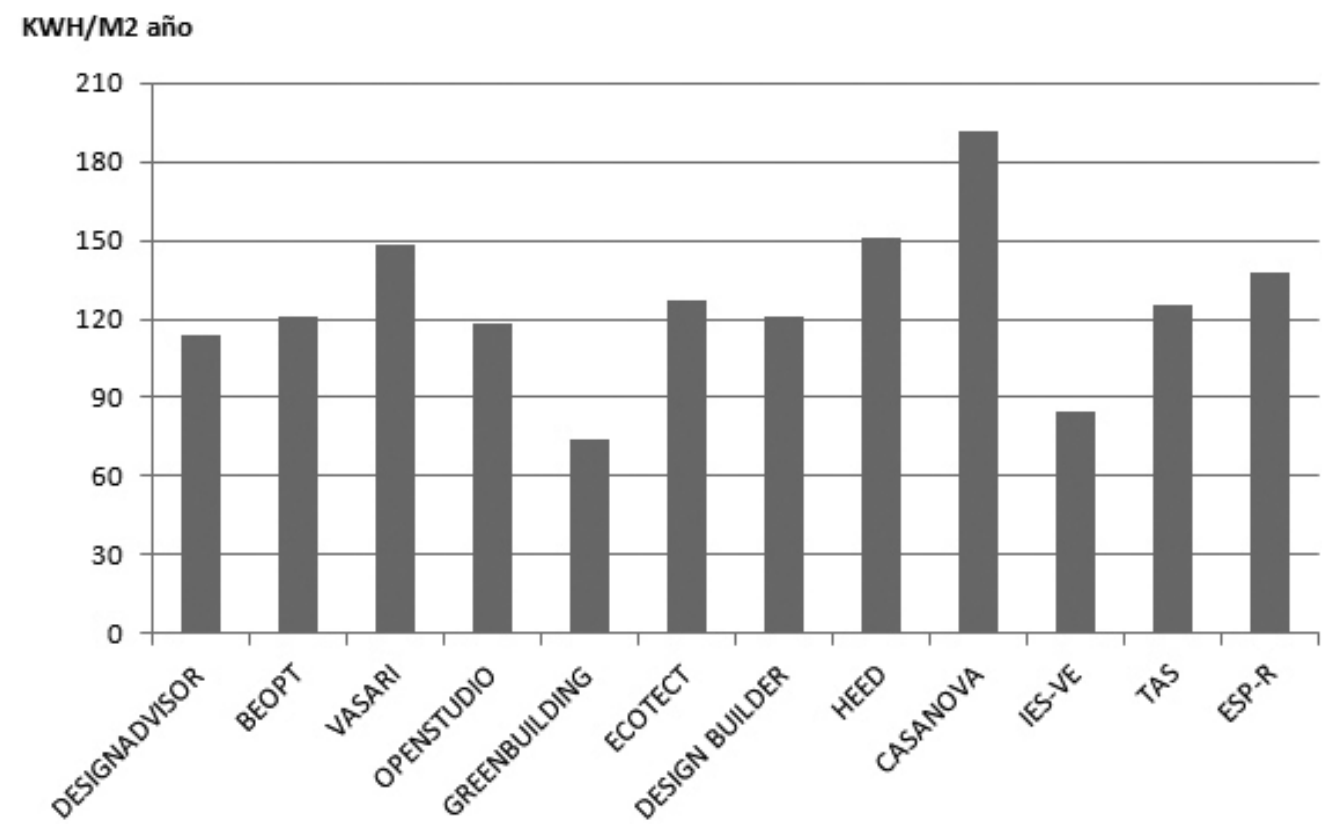

Figura 4. Resultados de Demanda con cada Programa.

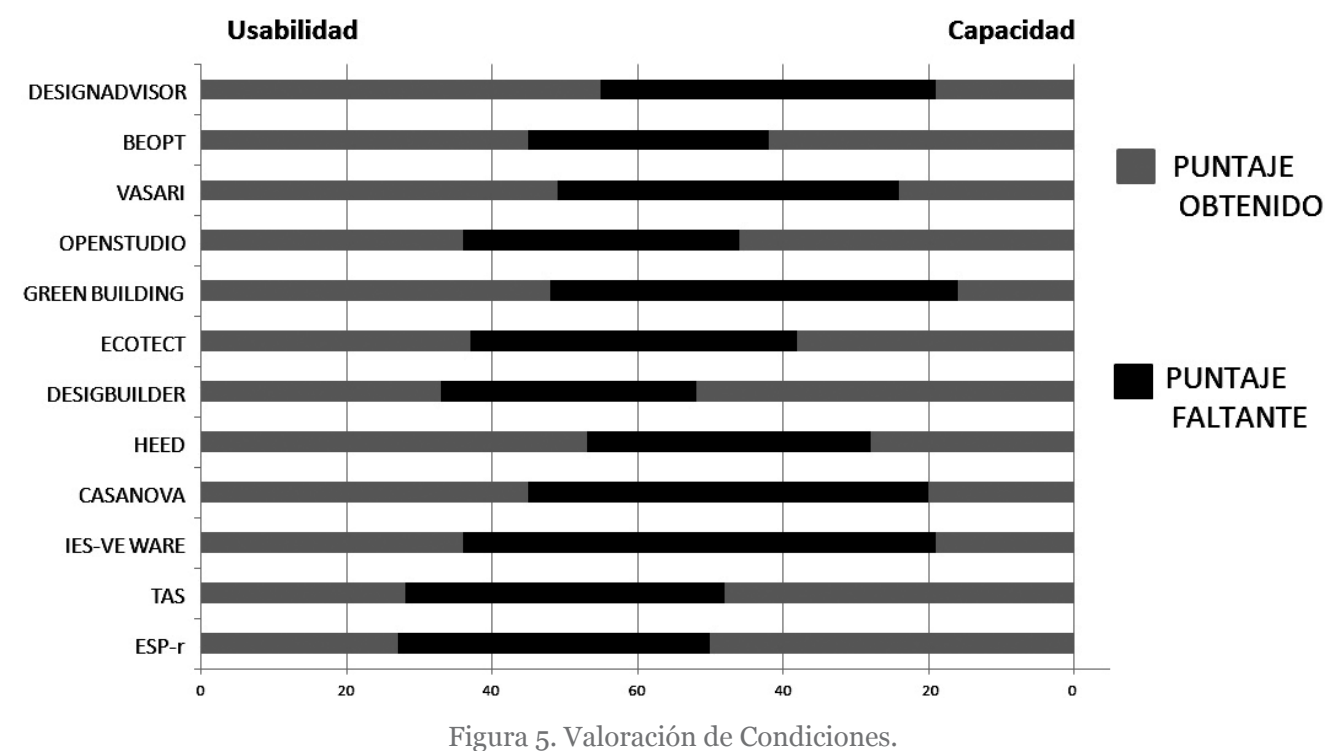

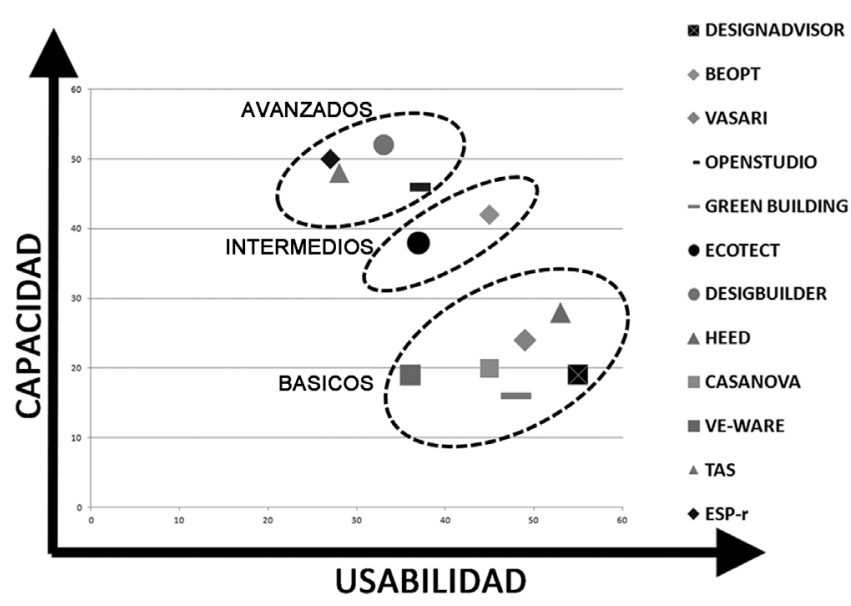

Figura 6. Síntesis comparativa de Capacidades/Usabilidad. variedad de análisis, precisión de los datos, adscripción a estándares y transferencia de archivos.

\subsection{Clasificación de Sistemas según Condiciones}

En la tabulación global se advirtieron diferentes tendencias entre los sistemas (Figura 6). Programas como Heed, Vasari, y servicios web como DesignAdvisor y Green Building, se caracterizan por su rapidez de trabajo, bajo costo o gratuidad, aunque también por una menor cantidad de aspectos analizados. Es decir con una alta usabilidad, pero escasas capacidades, por lo que podemos considerarlos como más «básicos». Aunque estos programas permiten que, conociendo sólo algunos conceptos, con un equipo doméstico y en poco tiempo (a veces en unos minutos), se puedan analizar desempeños energéticos generales de una vivienda. Algunas dificultades de operación (como conversión de unidades o falta de ante- 
cedentes), no se pueden subsanar rápidamente, pero se reducen con el uso reiterado del programa.

Por otro lado, los programas con mayores capacidades, que podemos denominar más «completos», como ESP-r, TAS y DesignBuilder, se distinguen por su mayor nivel de detalle en la modelación, descripción de equipamientos, horarios, archivos climáticos, diversidad de análisis, comprobación de normativas y relación con otros software. Con una buena asistencia, modificación de datos y representación de resultados. Pero también por un precio más alto, requerir mayores equipos y conocimiento especializado, mas antecedentes y tiempo de trabajo (varias horas o días para analizar una vivienda).

Algunos sistemas más recientes, como Ecotect, Ve-Ware y Beopt, podemos considerarlos como más balanceados o «intermedios», ya que combinan una variedad de capacidades, con una operación relativamente sencilla, precio intermedio y requerir un dominio general del tema. Permitiendo en pocas horas un estudio energético global de una vivienda.
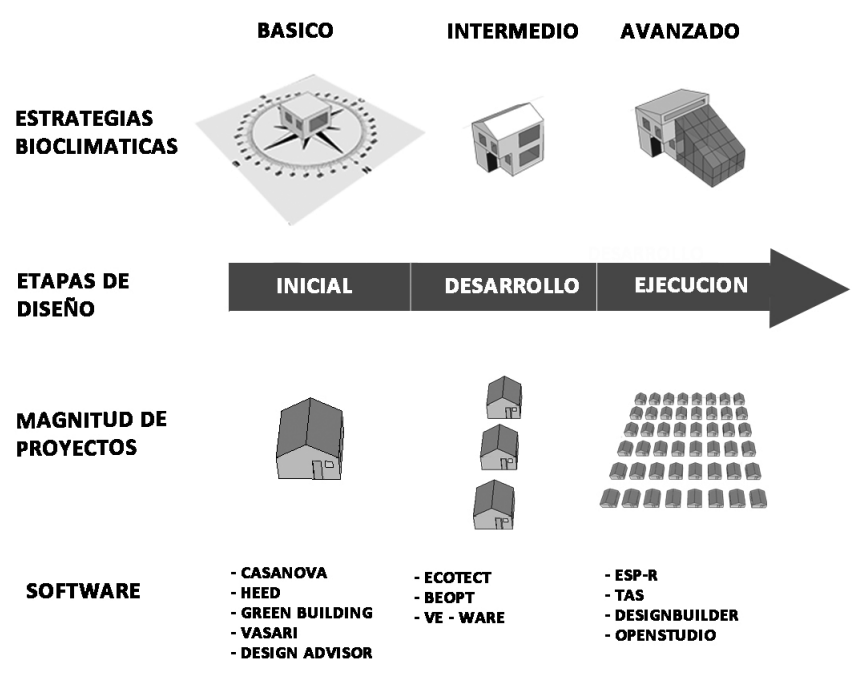

Figura 7. Aplicación de los distintos sistemas por rango.

\subsection{Aplicación de Sistemas}

Este panorama revela una variedad diferenciada de sistemas disponibles para estudios energéticos de edificación, que pueden utilizarse en diferentes condiciones o fases de diseño (13) (14). Como para viviendas unifamiliares deben ser particularmente eficaces, son más pertinentes los programas básicos. Los que pueden ser utilizados por cualquier profesional, en proyectos independientes y desde las fases iniciales del diseño, principalmente revisando aspectos globales de la construcción. Sin embargo algunas capacidades particulares, por ejemplo la simulación dinámica, datos climáticos, detalles de materiales, ocupación o geometría, que analizan mas cabalmente sistemas mayores, pueden requerirse en ciertas localizaciones (ciudades pequeñas o zonas rurales), de amplia oscilación diaria, con sistemas constructivos o formas especiales. También condiciones de certificación o relación profesional pueden inducir la utilización de algunos programas. Climas cálidos, estrategias bio-climáticas pasivas, así como equipos de climatización complejos deben ser estudiados con software avanzados.

De modo que progresivamente se debe considerar la utilización de sistemas con capacidad y utilización adecuada, que requieren más formación, implementación y tiempo de trabajo (Figura 7). Lo que se compensa con mayor frecuencia de utilización o magnitud de viviendas. Considerando el estudio de mejoramientos específicos del diseño durante su formulación, o en tipologías definidas.

En etapas avanzadas de desarrollo y en acciones masivas, especialmente de edificaciones existentes, se deben considerar sistemas completos. Los que permiten estudiar características detalladas en situaciones diferentes, revisando componentes o sistemas particulares y su variedad de impactos ambientales. Aunque también en algunas casas singulares por su relevancia social o experimental. Apoyando repercusiones relevantes en los desempeños energéticos.

Normalmente decisiones globales de forma o materialidad pueden lograr amplios mejoramientos, estudiando rápidamente grandes modificaciones con sistemas básicos. Pero también alteraciones constructivas menores o equipamientos especiales pueden generar reducciones masivas. Un software balanceado como BEOPT permite estudios acumulativos y optimización económica. Lo que no considera el popular software Ecotect, sin embargo éste se integra más fácilmente en los proyectos de edificación por su apoyo comercial y presentación gráfica. Del mismo modo, los software básicos son atractivos para los arquitectos, y los más completos, son más comprensibles para los ingenieros de especialidades.

De modo que las condiciones de los sistemas de simulación energéticas establecen variadas opciones para el análisis de viviendas unifamiliares. Promoviendo la utilización de programas básicos para estudios individuales preliminares, sistemas intermedios para una progresión y proyección de los mejoramientos, y más avanzados para desarrollos masivos y especializados.

\section{ANTECEDENTES RELEVANTES PARA LA SIMULACIÓN ENERGÉTICA}

Varios análisis energéticos de edificios (11) (12) (15), han planteado que los datos iniciales inciden significativamente en los resultados. Por esta razón revisamos los antecedentes requeridos en los distintos sistemas de simulación, y su influencia en diversos casos locales. En estudios de viviendas experimentales con climatización pasiva o mejoramientos de muros, análisis comparativo de tipologías y estándares más eficientes (16) (17) (18) (19) (20) (21) (22) (23) (24). Además de simular una docena de casas existentes, registrando sus datos reales para compararlos con la modelación respectiva. Realizando sucesivos ajustes para verificar su incidencia en los resultados. Identificando de este modo las adecuaciones y mejoramientos relevantes, que revelan condiciones significativas para estudios energéticos residenciales en la zona.

\subsection{Modelación}

En primera instancia, se advierten diferencias en las estrategias de modelación de los edificios. Los sistemas de simulación varían en sus operaciones y nivel de detalle requerido (Figura 8). Lo que incide en la facilidad de uso, y eventualmente, en la capacidad de análisis. Los programas más básicos ofrecen elementos volumétricos pre-definidos, en que se indican las medidas principales y se seleccionan características generales. Los más complejos utilizan coordenadas, trazados bidimensionales o tridimensionales de todos los recintos y vanos. 
Permiten definir distintas zonas y elaborar los elementos. Algunos programas avanzados apoyan esta tarea, integrando planos o geometrías de otros programas de diseño, o utilizando software gratuito y sencillo para modelar, como Sketchup. Aunque usualmente se debe modificar o complementar la información integrada (dividir zonas, establecer paramentos sin espesor), o eliminar detalles gráficos, y puede ser más efectivo modelarlos directamente. En edificios existentes se debe recopilar previamente la información, tomando sus medidas y características. Sin embargo, según las experiencias revisadas parece no ser relevante un registro detallado, bastan dimensiones generales consistentes. Al menos no varían mayormente los resultados de simulación en viviendas de la zona según los procesos de modelación. Sin embargo para mejoramientos específicos, que según algunos estudios (17) (20) (22) (24) pueden incidir hasta el 30\% de la demanda, se requiere una modelación detallada. Especialmente en la optimización volumétrica o de vanos, la adyacencia y obstrucción del entorno, la discriminación de zonas, la definición de áreas de captación y acumulación solar o sistemas de ventilación.
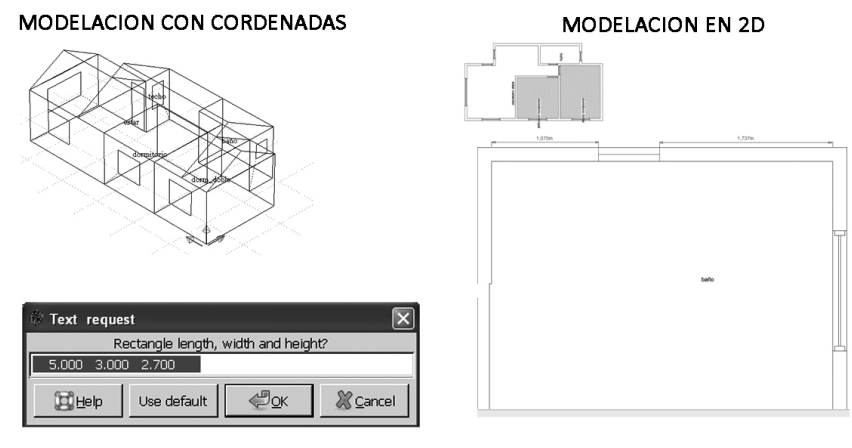

MODELACION EN 3D
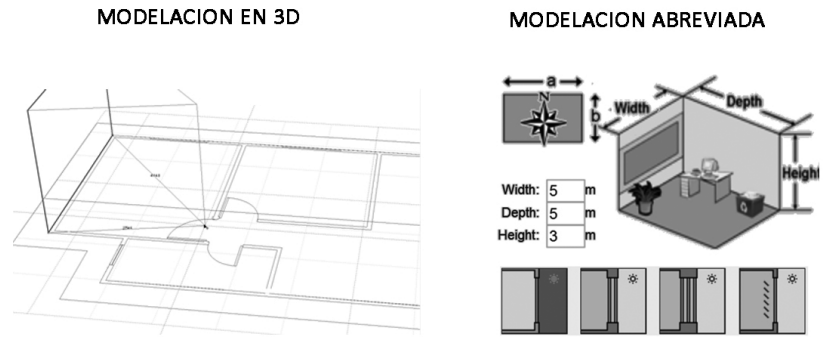

Figura 8. Estrategias de Modelación.

\subsection{Materiales y Equipamiento}

La descripción de materiales y equipamientos, y sus correspondientes propiedades energéticas, también es una actividad extensa y sujeta a interpretaciones según las categorías disponibles en los programas y los productos existentes en la zona. Especialmente relevantes son la transmitancia térmica de los distintos paramentos (que puede ser indicada o calculada), así como la eficiencia estimada de los equipos de climatización. De los cuales a veces se carece de información de los proveedores o de la ejecución real (por ejemplo, en espesores de materiales interiores en muros, desempeños de calefactores según el combustible utilizado o el aporte latente de equipos domésticos). Las consideraciones de equipamiento en los casos analizados en la zona, parecen tener una incidencia relevante, especialmente por los calendarios de calefacción (que llegan a influir hasta un 48\%), aunque las precisiones materiales son escasas. Mientras los mejoramientos sugeridos suelen destacar el aislamiento de la envolvente vertical (16) (18) (19) (20) (21) (23) aduciendo incidencias de hasta un tercio en el desempeño energético, y algunos mencionan también equipamientos más eficientes (21) (24), pero con una influencia más leve. Por tanto parece necesario discriminar cabalmente las características de estos elementos para un adecuado análisis energético.

\subsection{Calidad Constructiva}

También la mayoría de los programas generaliza algunos aspectos como los puentes térmicos, la cantidad de humedad en los materiales y la renovación de aire en los recintos, producto de las infiltraciones. Estas características son muy dependientes de las calidades constructivas locales, y en general se desconoce cabalmente su incidencia en varias tipologías y regiones, pero algunos estudios advierten incidencias altas en el comportamiento energético de las edificaciones (25) (26) (27) (28). En mediciones realizadas en viviendas en la región y en los casos revisados, se reconoció una variación relevante en el desempeño ambiental (en una media del 62\%), especialmente por permeabilidad de la envolvente, que depende del tipos de ventanas, sellado de puertas, ejecución de paramentos o exposición a vientos locales, en comparación con los valores inicialmente simulados. Un par de estudios de mejoramiento en la zona también mencionan influencias relevantes (18) (23). Aunque sin ponderar específicamente sus características, en cuanto a determinar cabalmente las magnitudes de infiltración según los tipos de edificación, por lo que en las simulaciones se utilizan estimaciones globales. Pero se advierte que es un factor sustancial que debe ser especialmente revisado, mediante mediciones en terreno (blower-door test) en construcciones existentes o estableciendo clasificaciones para nuevos edificios, para desarrollar estudios fiables en este aspecto.

\subsection{Ocupación}

Las características de ocupación usualmente son difíciles de registrar por su variabilidad en el tiempo, pero en edificios residenciales o de trabajo hay hábitos frecuentes. Los programas contemplan registros horarios por recintos, y características de actividades y vestimenta, aunque a veces resultan difíciles de interpretar. También los muebles, cortinas, revestimientos y colores, así como control de aberturas han relevado incidencias en el comportamiento ambiental. En los casos revisados se advirtieron diferencias en los periodos de ocupación declarados por recintos, que estimaban más de la mitad del tiempo efectivamente registrado en las viviendas, sin embargo estos ajustes expresaron luego una baja incidencia en las demandas (cerca del 5\%), mientras los estudios de mejoramientos no mencionan estos aspectos. Monitorizaciones regulares han demostrado también una relevante discrepancia entre lo declarado con las situaciones reales (29), por lo que parece necesario una observación meticulosa de las realidades simuladas, así como un cálculo detallado según habitaciones y horarios. Además el mejoramiento de las condiciones energéticas materiales de las viviendas, redundara luego en una mayor incidencia de los comportamientos personales.

\subsection{Clima}

Los archivos climáticos también son un antecedente de los análisis energéticos de edificios que presenta diversas consideraciones. La mayoría de los software utilizan datos climáticos provenientes de grandes centros urbanos (especialmente del hemisferio norte), y reconocen archivos en diferentes formatos (Figura 9). Los datos pueden ser plurianuales, de 
años típicos o días representativos e incluyen oscilación de temperaturas, humedad, radiación, velocidad del viento, etc. Las fuentes más utilizadas son los servidores Meteonorm y Weather Analytics con formatos TMY y TMM, y el EERE (del Departamento de Energía de Estados Unidos) con formato IWEC, como también se pueden crear archivos climáticos a partir de una estación meteorológica. Algunos estudios (15) (30) (31), además de comparaciones que realizamos entre archivos climáticos de distintas fuentes y registros meteorológicos de la zona, han revelado diferencias del $25 \%$ en los resultados de estudios energéticos de edificios, recomendando fundamentalmente utilizar datos IWEC con promedios superiores a quince años. También las condiciones locales de ángulo solar y radiación reflejada parecen tener incidencias relevantes. Igualmente la temperatura del suelo es asumida en muchos sistemas como similar al entorno, y suele tener unos pocos grados de diferencia que influyen en los resultados (15). Se ha discutido así mismo la implicancia del cambio climático en estos estudios, que deben predecir comportamientos futuros, en base a registros pasados. Aunque se desconocen cabalmente las alteraciones esperadas del calentamiento global en muchas regiones del planeta, se ha pronosticado una influencia moderada del cambio climático en el comportamiento ambiental de las edificaciones (30). Más reducida que la variación estacional o diaria, e inferior a los efectos locales que produciría el propio desarrollo energético y residencial. Tampoco se conocen usualmente muchas condiciones de micro-clima urbanos o del entorno residencial, que en viviendas unifamiliares y barrios habitacionales pueden ser relevantes. En este sentido conviene atender la base climática utilizada y consultar la situación particular en relación con los centros urbanos, para revisar ajustes específicos.

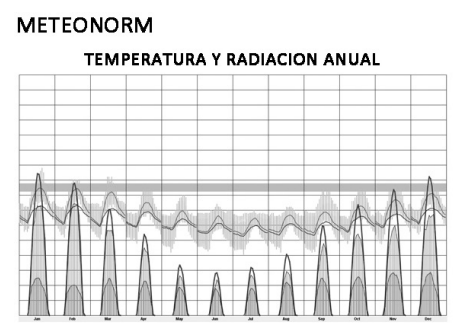

IWEC

TEMPERATURA Y RADIACION ANUAL
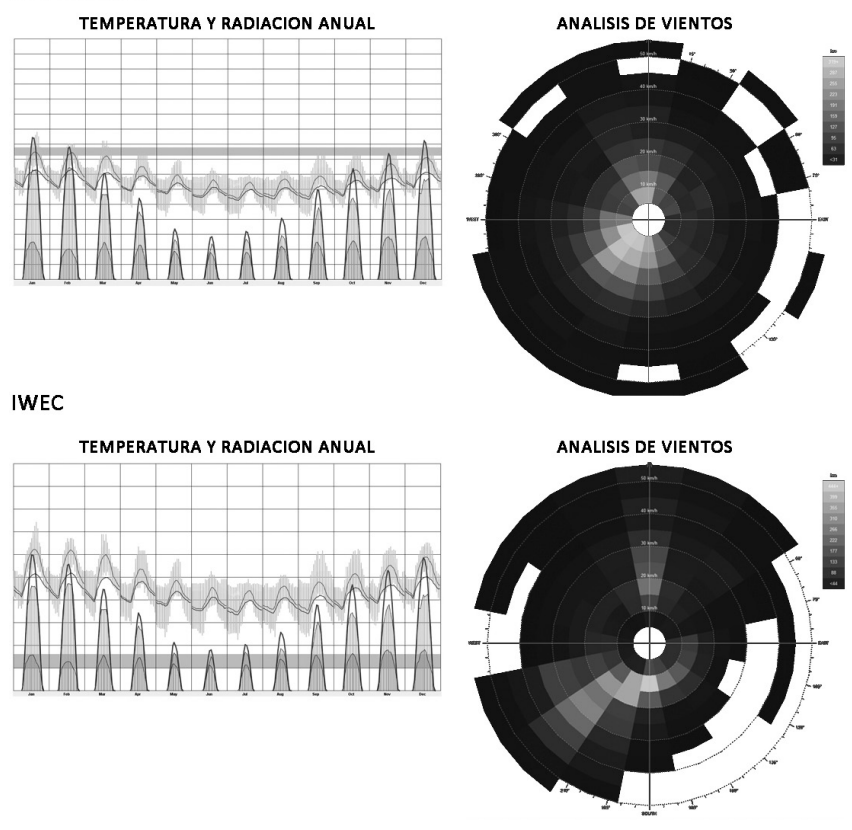

Figura 9. Comparación de fuentes de datos climáticos.

\subsection{Rango de Confort}

Un aspecto central en el análisis energético es la condición de confort estimada, que actúa de referencia para la revisión de demandas y desempeños. Aunque existen estándares internacionales, se ha advertido ampliamente sobre la incidencia de factores personales o culturales (32) (33). En estudios locales (34) (35) y en las monitorizaciones y encuestas de las viviendas revisadas en la zona se han comprobado requerimientos inferiores a los estimados globalmente (temperaturas monitoreadas menores a las bandas de confort, con índices de satisfacción térmica adecuadas), aunque revelando también diferencias de expectativas según nivel etáreo y género dentro del grupo familiar, combinada con distinciones por orígenes y nivel socioeconómico. También se ha encontrado una progresión en relación al desarrollo productivo, en que cada vez se exige mejores niveles de ambientación (35). Del mismo modo, esta consideración afecta la dotación de equipos de las viviendas, que están cambiando y mejorando, así como la calidad material y magnitud de las construcciones residenciales. Igualmente los costos y estrategias de generación, distribución y consumo energético pueden variar sustancialmente, especialmente en la situación chilena de alta dependencia exterior y matriz carbonizada. En este sentido se deben establecer situaciones de referencia, eventualmente modificables según la evolución cultural.

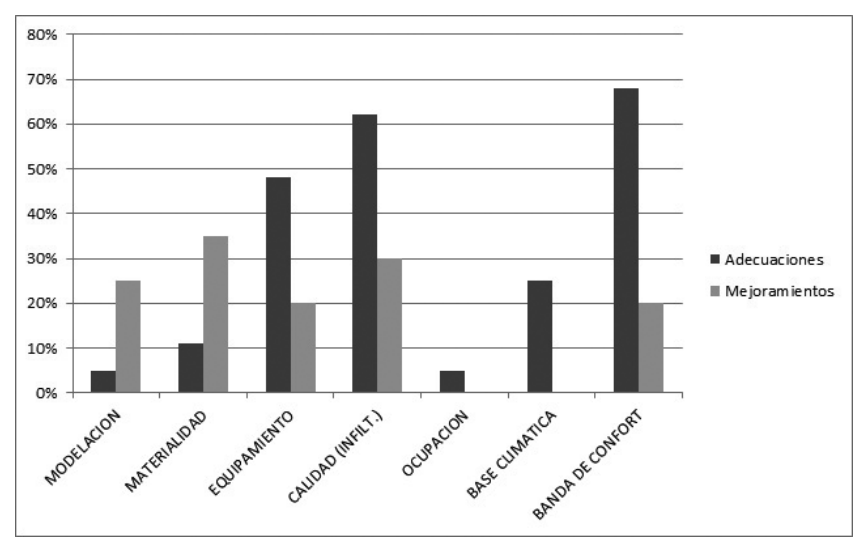

Figura 10. Incidencias de Antecedentes en Resultados

En resumen, sobre los antecedentes requeridos para un análisis energético de vivienda unifamiliar, a partir de los aspectos mencionados en estudios en la zona y la comparación de simulaciones de viviendas existentes con registros en terreno, parece relevante elaborar una modelación básicamente adecuada, con una revisión de materiales y equipamientos, atender la ocupación y seleccionar un archivo climático adecuado, considerando especialmente los factores de calidad de construcción y confort esperado (Figura 10).

\section{ANÁLISIS DE RESULTADOS}

La información resultante de los software de simulación energética puede ser de variada índole, principalmente valores de demandas de energía en relación a un rango de confort, para distintos periodos temporales (anuales, estacionales o diarios), condiciones, fuentes, cargas, zonas. En distintas unidades de medida o relaciones con la superficie construida u ocupantes del edificio, en tablas numéricas o gráficos de barras. También valores de $\mathrm{CO}_{2}$ producidos por periodos; flujos de energía según partes del edificio; valores o gráficos de radiación solar; iluminación natural en los recintos, consumos de agua o combustibles; comprobación de estándares, asoleamientos del volumen construido en distintos momentos del día o el año, en diferentes puntos de vista, etc. En algunos se pueden revisar representaciones de movimiento de aire, variaciones de temperatura o humedad en los elementos constructivos. 


\subsection{Tipos de Análisis}

Estos resultados pueden ser provechosos para definir certificaciones, comparar con referencias, revisar alternativas, desarrollar mejoramientos individuales o globales. Algunos permiten integrar costos, efectuar comparaciones con un caso base o entre varias simulaciones consecutivas. Modificando valores o elementos específicos, lo que se denominan «análisis de sensibilidad» (36). Por ejemplo, revisar si la magnitud de un aislamiento permite reducir significativamente la demanda, o si una cantidad de ocupación corresponde a un valor de temperatura monitoreado, para calibrar el modelo. Esto requiere ordenar las secuencias de simulaciones, almacenar resultados y realizar tablas o gráficos de las alternativas.

Cuando se combinan dos o más valores independientes en un análisis multi-factorial, usualmente dirigidas a revisar influencias diferentes o relación entre distintos valores de antecedentes y condiciones, realizando una cantidad creciente de simulaciones, se denominan «análisis paramétricos» (37). Lo que requiere definir el ingreso de datos, como su procesamiento y revisión, a veces implementadas computacionalmente. En algunos casos con precios de materiales o equipos y estimaciones de retorno de inversión, buscando el valor más reducido o más efectivo, lo que se denomina «análisis de optimización» (38). Aunque en las construcciones, estas modificaciones pueden ser muy diversas, y desarrolladas de distintas maneras.

En el estudio de alternativas, se suelen realizar flujogramas de las condiciones, tablas de variación y/o gráficos de síntesis (Figura 12). En ocasiones se pueden utilizar métodos estadísticos de análisis diferencial o estrategias probabilísticas para reducir la cantidad de simulaciones, como el método de Montecarlo. Lo que puede ser implementado con algunas utilidades como EP-macro, EZ-Plus, COMFEM, JePlus; Genopt, Beopt o Design Builder que utilizan EnergyPlus como «motor de cálculo». En algunos casos (como Genopt) se implementan algoritmos genéticos seleccionando datos con estrategias evolutivas. Aunque aparentemente una estimación intuitiva de alternativas adecuadas parece ser más efectiva en edificaciones pequeñas, como las viviendas.

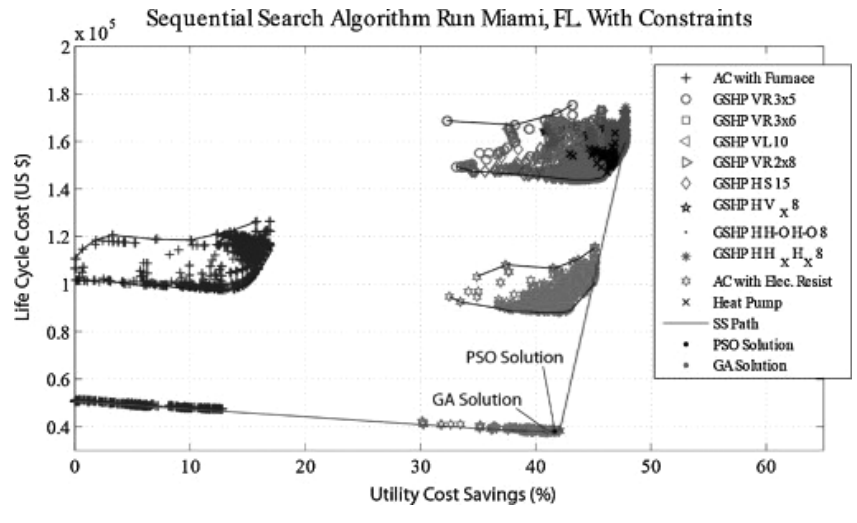

Figura 12. Análisis de Optimización (BEOPT).

Algunas alternativas pueden implicar variaciones geométricas, que se pueden preparar en un software de diseño con programación paramétrica de formas y enviarlas directamente al análisis (como Grasshopper-Rhino y Ecotect a través de utilidades como Geco). En una estrategia de «diseño paramétrico» para determinar formas óptimas para ciertas condiciones (Figura 11).

Las capacidades de visualización volumétrica también son relevantes para revisar condiciones cualitativas, ya sea estéticas o culturales. Considerando además que las modificaciones formales suelen tener altas incidencias en los desempeños energéticos, especialmente en construcciones de pequeño tamaño, como las residenciales. De modo que las presentaciones del modelo, con sus alteraciones, así como la integración con otros programas de diseño arquitectónico o visualización avanzada, son importantes para ampliar las condiciones de estudio y sus implicancias. Incluyendo también la resolución constructiva de las modificaciones, que se facilitan con la vinculación de información (transferencia de archivos) con los sistemas de modelación y gestión constructiva, como los software BIM (building information modeling). Los cuales también pueden contribuir en la digitalización inicial de la simulación.

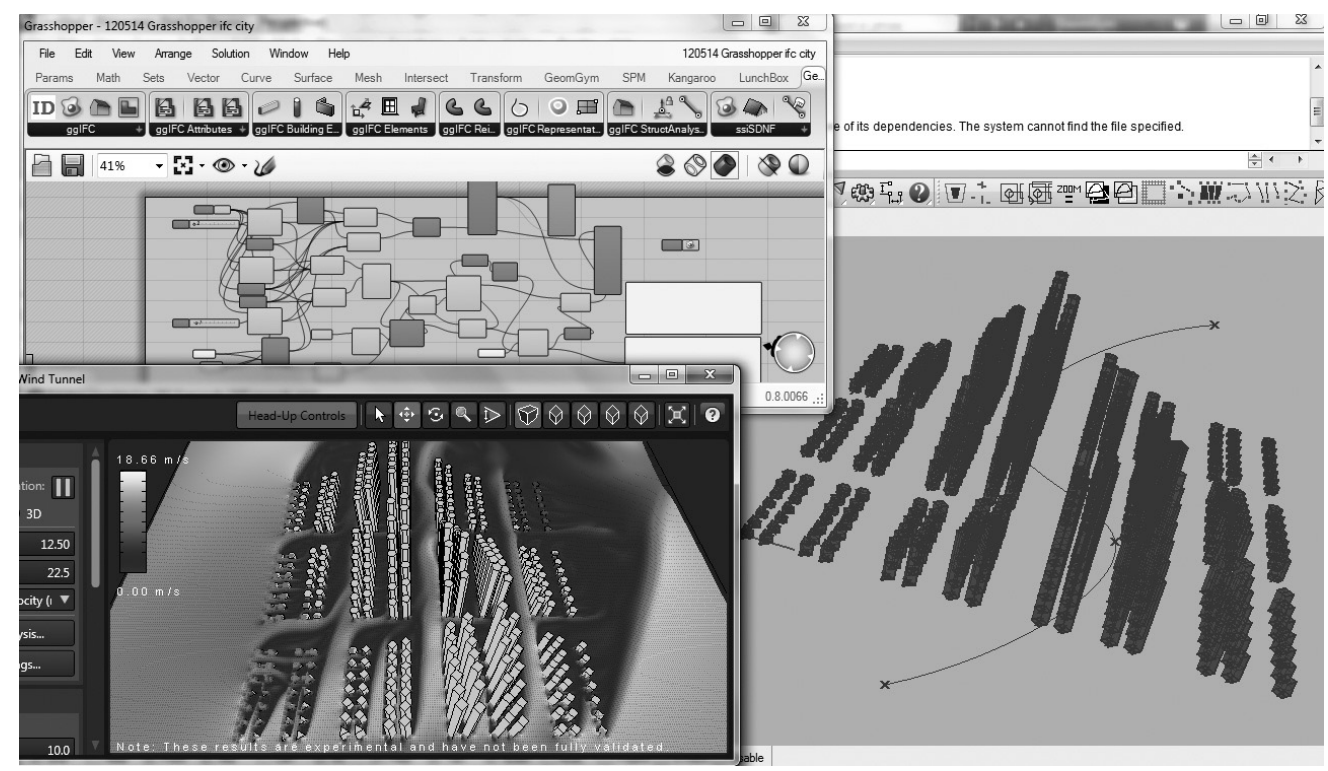

Figura 11. Diseño Parmétrico con Análisis Energético. Fuente: http://geometrygym.blogspot.com 


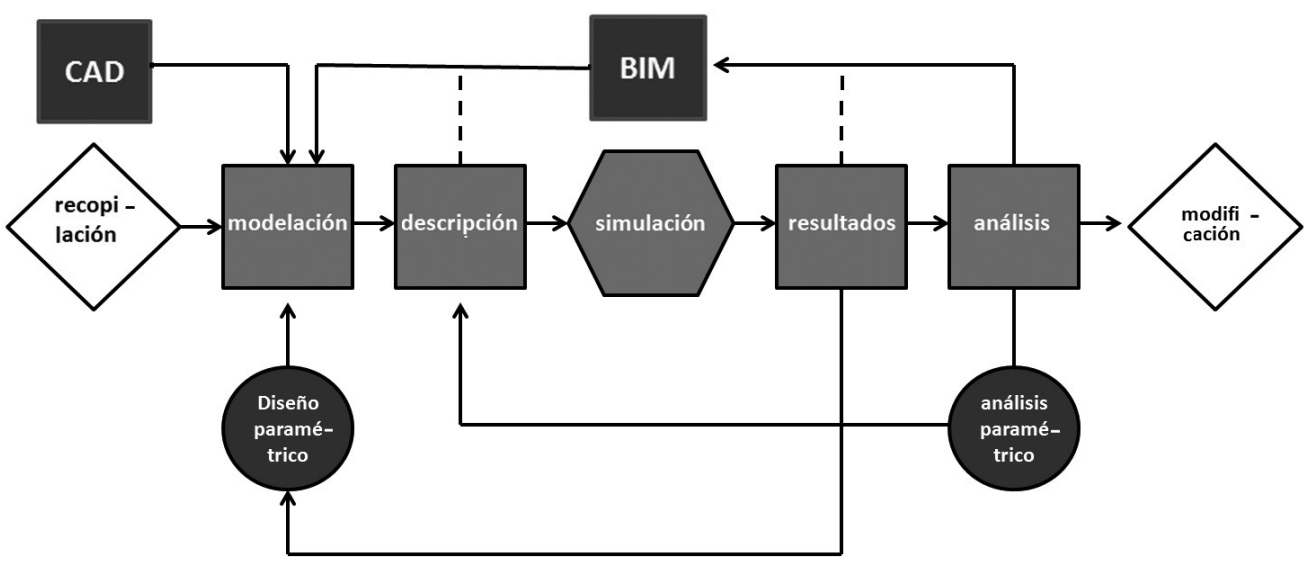

TIEMPOS DE TRABAJO

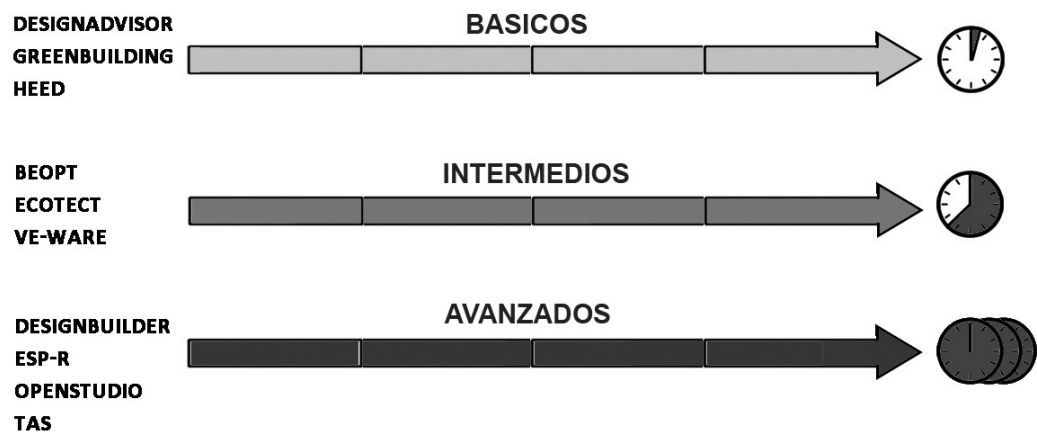

Figura 13. Proceso General de Simulación Energética.

\subsection{Proceso general}

La simulación energética está constituida entonces por una secuencia de tareas, centradas en una operación de cálculo, que requiere previamente una modelación y descripción (de acuerdo ciertos antecedentes), y subsecuentemente produce resultados y análisis (Figura 13). Lo que implica acciones previas de recopilación, y posteriormente de modificación de la edificación, a la vez que se puede relacionar con otras capacidades de análisis o diseño. Lo cual puede ser realizado con el mismo o varios software, conformando entonces una variedad de actividades mayormente apoyadas digitalmente. Los sistemas de simulación, permiten realizar las tareas centrales, especialmente en vivienda, en periodos muy diferenciados de acuerdo a sus capacidades y usabilidad (básica, intermedia y avanzada), pero a la vez con diversas aplicaciones e implicancias en el desarrollo residencial.

\subsection{Experiencias de Mejoramientos Energéticos}

Diversas experiencias de análisis energético de viviendas en la zona (16) (17) (18) (19) (20) (21) (22) (23) (24), mencionan haber realizado simulaciones con diferentes software, en varias alternativas (en alguno con millares de simulaciones usando programas estadísticos). Con resultados de demanda energética, y ocasionalmente de asoleamiento, iluminación o conductividades específicas. Determinando modificaciones de volumetría, orientación, distribución, apertura de vanos, instalación de equipos, y principalmente mejoramientos de envolventes verticales exteriores, que plantean reducciones significativas de consumo, mejor confort y desempeño sustentable de las viviendas. Con estudios de costos asociados y recuperaciones económicas, aunque rara vez con verifica- ciones de su ejecución. En la mayoría de los casos, logrando integrar las sugerencias en el proceso de proyecto, y en varias ocasiones realizando las medidas definidas, en algunos comprobando mejoramientos de consumo y calidad ambiental, aunque menores a las estimadas.

\section{CONCLUSIONES}

Este trabajo ha identificado sistemas para simulación energética de edificios, revisando condiciones de utilización y capacidades técnicas de una docena de programas o servicios web, especialmente para el estudio de viviendas aisladas. Se ha comprobado una variedad de herramientas disponibles, con un nivel creciente de detalle y requerimientos. Desde sistemas básicos que se pueden ocupar fácilmente, programas intermedios que balancean capacidades y funcionalidad, hasta sistemas avanzados, para estudios más especializados y de alcance amplio, que se pueden utilizar en distintas fases del proyecto, aspectos de estudio y magnitudes de aplicación. Por lo que se sugiere que la selección del software apropiado para el análisis energético de residencias unifamiliares, depende de la fase y extensión de su utilización, y por ende, la implementación requerida, la duración del estudio y características analizadas. Disponiendo de distintas alternativas de programas, que se pueden elegir por facilidades locales (distribuidores, centros de enseñanza o colegas experimentados).

También se han identificado antecedentes de modelación y materialidad, y en especial de la ocupación y clima, que deben estar cabalmente definidos para una simulación adecuada. Revisando en esta región especialmente la infiltración y estimaciones de confort. Se sugiere considerar también el análisis de los resultados, en cuanto a su compatibilidad con 
las consideraciones arquitectónicas, constructivas y económicas, mediante estrategias de visualización y comparación numérica para identificar aspectos efectivamente relevantes y modificables en el comportamiento energético. Con el fin de determinar mejoramientos fiables y efectivos de la calidad ambiental de las viviendas, en procesos de simulación con un nivel creciente de detalle. Atendiendo condiciones formales, constructivas y climáticas zonificadas, con posibilidades de revisión iterativa y financiera, que pueden redundar en desarrollos significativos del desempeño energético residencial.

\section{AGRADECIMIENTOS}

Proyecto Fondecyt 1120165 y a los profesionales entrevistados.

\section{REFERENCIAS}

(1) Ministerio de Energía de Chile. (2012). Balance Nacional de Energía 2011. Santiago.

(2) U.S. Department of Energy. Building Energy Software Tools Directory. http://apps1.eere.energy.gov/buildings/tools_ directory.

(3) ISO. (2008). ISO 13790:2008-o9: Thermal performance of buildings-Calculation of energy use for space heating and cooling. International Organization for Standardization.

(4) ASHRAE. (2007). Standard 90.1. American Society of Heating, Refrigerating and Air-Conditioning Engineers, Atlanta, GA.

(5) IDAE. (2008). Guía Técnica, Procedimientos y Aspectos de la Simulación de Instalaciones Térmicas en los Edificios, Madrid (España). Ministerio de Industria, Turismo, y Comercio.

(6) Jennings, J., Lam, K.P., Huang, Y., Zhai, C. (2004). Energy Modeling's Tools Assessment for Early Design Phase. Pittsburgh: Center for Building Performance and Diagnostics at Carnegie Mellon University.

(7) Crawley, D., Hand, J., Kummert, M., Griffith, B. (2008). Contrasting the capabilities of building energy performance simulation programs. Building and Environment, 43(4): 661-673, doi: http://dx.doi.org/10.1016/j.buildenv.2006.10.027.

(8) Granlund, O., Korhonen M., Laine T. (2008). Energy Analysis Software Evaluation, BIM Interface and Interoperability. Granlund Corp.

(9) Attia, S., Beltrán, L., De Herde, A., Hensen, J. (2009, 27-30 de julio). Architect friendly: A comparison of ten different building performance simulation tools. En XI IBPSA Building Simulation Conference, 49: 2-15. Glasgow, UK.

(10) Judkoff, R., Wortman, D., O’Doherty, B., Burch, J. (2008). A Methodology for Validating Building Energy Analysis Simulations. Golden, CO (USA): Solar Energy Research Institute (now NREL), SERI/TR-254-1508.

(11) Ljungberg, A., Jansson, J. (2010). Uncertainties in energy calculations and their effect upon the results of theoretical specific energy usage (Master of Science Thesis). Göteborg, Sweden: Chalmers university of Technology.

(12) Palme, M. (2010). La sensibilidad energética de los edificios (Tesis doctoral). Barcelona: Universidad Politécnica de Cataluña.

(13) Ministerio de Vivienda y Urbanismo de Chile. (2010). Informe Urbano Habitacional. Período 20o6-20o9. Comisión de Estudios Habitacionales y Urbanos, Santiago.

(14) Morbitzer, C., Strachan, P., Webster, J., Spires, B., Cafferty, D. (2001, 13-15 de agosto). Integration of Building Simulation into the Design Process of an Architecture Practice. En Seventh International IBPSA Conference, pp. 697-704. Rio de Janeiro, Brazil.

(15) Purdy, J., Beausoleil-Morrison. I. (2001, 13-15 de agosto). The significant Factors in Modelling Residential Buildings. En Seventh International IBPSA Conference, pp. 207-214. Rio de Janeiro, Brazil.

(16) Müller, E. (2000, 10-14 de octubre). Estudios Paramétricos con Simulaciones Térmicas para Viviendas con Climatización Pasiva en la Zona Central de Chile. En IX Congreso Chileno de Ingeniería Mecánica. Valparaíso, Chile.

(17) Trebilcock, M., Burdiles, R., Fissore, A. (2001, 21-23 de noviembre). La modelación y simulación energético-ambiental como herramienta de rediseño arquitectónico. En Congreso Sigradi 2001, pp. 83-86. Concepcion, Chile.

(18) Vera, S., Ordenes, M. (2002). Evaluación del desempeño Energi-térmico de una vivienda social en Chile, utilizando un programa de simulación energética de edificios. Revista Ingeniería de Construcción, 17(3): 133-142.

(19) Bustamante, W., Bobadilla, A., Navarrete, B., Saelzer, G., Vidal,Sergio (2005). Uso eficiente de la energía en edificios habitacionales. Mejoramiento térmico de muros de albañilería de ladrillos cerámicos. Revista de la Construcción, 4(2): 5-12.

(20) Roldán, J., Díaz, B., Andrade, P., Castro, C., Gutiérrez, C., Uribe ,S. (2008). Conjunto de Viviendas Bioclimáticas Socialmente Sustentables en Santiago de Chile: Propuesta de un Modelo con Crecimiento Interior. Avances en Energías Renovables y Medio Ambiente, Vol. 12. Argentina.

(21) Bustos, L., Kairies, D. (2008). Reglamentación Térmica de la Vivienda Social en Chile. Seminario de Título en Arquitectura. Universidad del Bío-Bío.

(22) Celis, F., Garcia, R., Trebilcock, M., Escorcia, O., Bruscato, U., Diaz, M. (2011). Análisis energético de las viviendas del centro-sur de Chile. Arquiteturarevista, 8(1): 62-75, doi: http://dx.doi.org/10.4013/arq.2012.81.07.

(23) Escorcia, O., Garcia, R., Trebilcock, M., Bruscato, U. (2012). Mejoramientos de envolvente para la eficiencia energética de viviendas en el centro-sur de Chile. Informes de la Construcción, 64(528): 563-574, doi: http://dx.doi.org/10.3989/ic.11.143.

(24) Carrasco, J., Kokogiannakis, G. (2012). Feasibility of PassivHaus standards and alternative passive design on climatic zones of Chile - Determination of energy requirements with dynamic simulation. Habitat Sustentable, 2(1): 59-71.

(25) Martin, K., Erkoreka, A., Flores, I., Odriozola, M., Sala, J.M. (2011). Problems in the calculation of thermal bridges in dynamic conditions. Energy and Buildings, 43(2-3): 529-535, doi: http://dx.doi.org/10.1016/j.enbuild.2010.10.018.

(26) Erazo, R. (2007). Variación de la conductividad térmica con la humedad en materiales de construcción (Memoria para optar al título de ingeniero civil). Universidad de Chile.

(27) Kronvall, J. (1978). Testing of homes for air leakage using a preasure method. ASHRAE Transactions 84(1): 72-79.

(28) Persily A. (1982). Understanding Air Infiltration in Homes. Report No. 129, Center for Energy and Environmental Studies, Princeton, NJ. Princeton University. 
(29) León, A., Muñoz, S., León J., Bustamante, P. (2010). Monitorización de variables medioambientales y energéticas en la construcción de viviendas protegidas: Edificio Cros-Pirotecnia en Sevilla. Informes de la Construcción, 62(519): 67-82. doi: http://dx.doi.org/10.3989/ic.09.045.

(30) Crawley, D. (2007). Estimating the Impacts of Climate Change and Urbanization on Building Performance. En Proceedings of Building Simulation, 'o7 Conference IBPSA. Beijing, China.

(31) Seo, D, Huang, Y, Joe, Y., Moncef, K. (2010). Impact of Typical Weather Year Selection Approaches on Energy Analysis of Buildings. ASHRAE Transactions, 116(1): 416.

(32) Brager, G.S., Dear, R. J. (1998). Thermal adaptation in the built environment: A literature review. Energy and Buildings, 27(1): 83-96, doi: http://dx.doi.org/10.1016/So378-7788(97)00053-4.

(33) Frontczak, M. (2011). Human comfort and self-estimated performance in relation to indoor environmental parameters and building features (Tesis doctoral). Lyngby: Technical University of Denmark.

(34) Sarmiento, P., Hormazabal, N. (2003). Habitabilidad térmica en las viviendas básicas de la zona Central de Chile, a la luz de los resultados preliminares del proyecto Fondef DooI103941. Revista INVI, 18(46): 23-32.

(35) Corporación de Desarrollo Tecnológico. (2010). Estudio de Usos Finales y Curva de Oferta de Conservación de la Energía en el Sector Residencial de Chile. Santiago: Cámara Chilena de la Construcción.

(36) Westphal, F., Lambert, R. (2005, 15-18 de agosto). Building Calibration Using Sensitivity Analysis. En Ninth International IBPSA Conference, pp.1331-1338. Montréal, Canada.

(37) O’Brien, W. T., Athienitis, K., Kesik, T. (2011). Parametric analysis to support the integrated design and performance modeling of net zero energy houses. ASHRAE Transactions, 117(1): 945-960.

(38) Christensen, C., Anderson, R., Horowitz, S., Courtney, A., Spencer, J. (2006, agosto). BEopt ${ }^{\mathrm{TM}}$ Software for Building Energy Optimization: Features and Capabilities. Technical Report NREL/TP-550-39929. USA: U.S. Department of Energy. 